\title{
Reproducibility Between Brain Uptake Ratio Using Anatomic Standardization and Patlak-Plot Methods
}

Takayuki Shibutani ${ }^{1}$, Masahisa Onoguchi ${ }^{1}$, Atsushi Noguchi ${ }^{2}$, Tomoki Yamada ${ }^{3}$, Hiroko Tsuchihashi ${ }^{3}$, Tadashi Nakajima ${ }^{3}$, and Seigo Kinuya ${ }^{4}$

\begin{abstract}
${ }^{I}$ Department of Quantum Medical Technology, Graduate School of Medical Sciences, Kanazawa University, Ishikawa, Japan; ${ }^{2}$ Department of Radiological Technology, Faculty of Health Sciences, Butsuryo College of Osaka, Osaka, Japan; ${ }^{3}$ Department of Radiological Technology, Kishiwada City Hospital, Osaka, Japan; and ${ }^{4}$ Department of Biotracer Medicine, Graduate School of Medical Science, Kanazawa University, Kanazawa, Ishikawa, Japan
\end{abstract}

The Patlak-plot and conventional methods of determining brain uptake ratio (BUR) have some problems with reproducibility. We formulated a method of determining BUR using anatomic standardization (BUR-AS) in a statistical parametric mapping algorithm to improve reproducibility. The objective of this study was to demonstrate the inter- and intraoperator reproducibility of mean cerebral blood flow as determined using BUR-AS in comparison to the conventional-BUR (BUR-C) and Patlakplot methods. Methods: The images of 30 patients who underwent brain perfusion SPECT were retrospectively used in this study. The images were reconstructed using ordered-subset expectation maximization and processed using an automatic quantitative analysis for cerebral blood flow of ECD tool. The mean SPECT count was calculated from axial basal ganglia slices of the normal side (slices 31-40) drawn using a 3-dimensional stereotactic region-of-interest template after anatomic standardization. The mean cerebral blood flow was calculated from the mean SPECT count. Reproducibility was evaluated using coefficient of variation and Bland-Altman plotting. Results: For both inter- and intraoperator reproducibility, the BUR-AS method had the lowest coefficient of variation and smallest error range about the Bland-Altman plot. Mean CBF obtained using the BUR-AS method had the highest reproducibility. Conclusion: Compared with the Patlak-plot and BUR-C methods, the BUR-AS method provides greater inter- and intraoperator reproducibility of cerebral blood flow measurement.

Key Words: brain perfusion SPECT; Patlak plot method; brain uptake ratio; reproducibility; cerebral blood flow (CBF)

J Nucl Med Technol 2015; 43:261-266

DOI: 10.2967/jnmt.115.162115

S everal different techniques have been applied for measurement of cerebral blood flow (CBF): xenon-enhanced CT, perfusion CT using contrast agents, MR imaging using arte-

\footnotetext{
Received Aug. 19, 2015; revision accepted Aug. 20, 2015.

For correspondence or reprints contact: Masahisa Onoguchi, Department of Quantum Medical Technology, Graduate School of Medical Sciences, Kanazawa

University, 5-11-80, Kodatsuno, Kanazawa, Ishikawa, 920-0942, Japan.

E-mail: onoguchi@staff.kanazawa-u.ac.jp

Published online Sep. 3, 2015.

COPYRIGHT (C 2015 by the Society of Nuclear Medicine and Molecular Imaging, Inc.
}

rial spin labeling, SPECT, and PET (1-3). Perfusion CT and xenon-enhanced CT are not in common use, because the former is an invasive technique and the latter is available in only a few institutions. The MR imaging technique for this application requires a high magnetic field and proprietary software. It has not yet become a general examination. On the other hand, nuclear medicine examinations for the quantitative measurement of CBF have been widely performed using SPECT with ${ }^{99 \mathrm{~m}} \mathrm{Tc}$-hexamethylpropyleneamine oxime, ${ }^{99 \mathrm{~m} T c-L, L-e t h y l c y s t e i n a t e ~ d i m e r ~(99 m T c-E C D), ~ a n d ~} \mathrm{~N}$-isopropyl$p$-iodoamphetamine ( ${ }^{123} \mathrm{I}$-IMP) and using PET with ${ }^{15} \mathrm{O}-$ labeling agents. The PET technique using ${ }^{15} \mathrm{O}$-labeling agents is more accurate than the 3 SPECT agents. However, PET has some limitations in that it cannot be performed without a cyclotron and synthesis device. In addition, the quantitative measurement of SPECT counts is more accurate using ${ }^{123}$ I-IMP than using other agents; however, ${ }^{123} \mathrm{I}$-IMP SPECT has the drawback of requiring arterial blood sampling. Conversely, quantitative analysis using ${ }^{99 \mathrm{~m} T c-l a b e l i n g}$ agents is noninvasive and does not require arterial blood sampling.

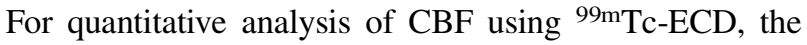
Patlak-plot and brain-uptake-ratio (BUR) methods have been reported (4-10). The Patlak-plot method is performed in quantitative analysis to calculate mean and regional CBF using SPECT data and radionuclide angiography ranges from the vertex to the aortic arch. In addition, the BUR method is applied in quantitative analysis to calculate mean and regional CBF using SPECT data and dynamic thorax data, where mean $\mathrm{CBF}$ is calculated from dynamic data before the Lassen process and regional CBF is calculated from SPECT data after the Lassen process $(4-6,10)$.

It has been reported that the Patlak-plot method has mean and regional CBF variations due to differences in intra- and interoperator reproducibility in such steps as the drawing of regions of interest (ROIs) over the aortic arch, brain, and normal basal ganglia; the determination of brain perfusion index; the setting of axes for SPECT image reconstruction; and the selection of slices for normal basal ganglia. Improvements have been proposed $(4,10-14)$. 
Mean and regional CBF variations due to the differences in intra- and inter- operator reproducibility have also been reported for the BUR method. Variability has been reported in such steps as the drawing of ROIs over the aortic arch and the normal basal ganglia, the $\gamma$-function fitting process for time-activity curves, the setting of axes for SPECT image reconstruction, and the selection of slices for normal basal ganglia. Improvements have also been proposed for this method $(4,10,11,15-17)$. These quantitative analysis errors may decrease diagnostic accuracy during follow-up and when determining the need for treatment adaptation. Therefore, achieving a reproducible $\mathrm{CBF}$ is important to maintaining diagnostic accuracy.

Takaki et al. reported that regional CBF reproducibility was improved by the use of SPECT images anatomically standardized with a statistical parametric mapping algorithm to automate the selection of slice ranges and basal ganglia ROIs in the Lassen process (12). However, anatomic standardization has not been applied to mean SPECT count, which is needed to calculate mean CBF with the BUR method. We defined BUR with anatomic standardization using the statistical parametric mapping algorithm (BUR-AS), which then analyzed mean SPECT count. The BUR-AS method is expected to improve the inter- and intraoperator reproducibility of mean CBF measurements. The objective of this study was to demonstrate this reproducibility for the BUR-AS method in comparison to the conventional BUR (BUR-C) and Patlak-plot methods.

\section{MATERIALS AND METHODS}

\section{Subjects}

Images for 30 patients (11 men and 19 women; age range, 25$88 \mathrm{y}$; mean age, $71 \mathrm{y}$ ) who underwent brain perfusion ${ }^{99 \mathrm{~m} T c-E C D}$ SPECT in 2013 were retrospectively used in this study. These subjects had encephalosis $(n=1)$, Parkinson disease $(n=6)$, moyamoya disease $(n=2)$, degenerative disease $(n=3)$, Alzheimer disease $(n=4)$, dementia with Lewy bodies $(n=2)$, frontotemporal dementia $(n=1)$, internal carotid artery stenosis $(n=2)$, internal artery stenosis occlusion $(n=3)$, dementia $(n=4)$, and cerebral infarction $(n=1)$. One subject had a normal brain. Permission for this study was obtained from the hospital ethics committee.

\section{Acquisition Protocols}

${ }^{99 \mathrm{~m}} \mathrm{Tc}-\mathrm{ECD}$ imaging was performed using a dual-head SPECT scanner (Infinia3; GE Healthcare). After a bolus injection of $600 \mathrm{MBq}$ of ${ }^{99 \mathrm{~m}} \mathrm{Tc}-\mathrm{ECD}$ into the right brachial vein, radionuclide angiography from the vertex to the aortic arch was performed for 2 min ( $1 \mathrm{~s}$ per frame; matrix, $128 \times 128$; zoom factor, 1.0 ; pixel size, $4.42 \mathrm{~mm}$ ) using 1 of the 2 detectors equipped with a lowenergy high-resolution parallel-hole collimator and a $140-\mathrm{keV} \pm$ $10 \%$ energy window. The SPECT study was performed using the same collimators and energy window. Projection data were acquired with a $64 \times 64$ matrix (zoom factor, 2.0; pixel size, $4.42 \mathrm{~mm}$ ) continuously over $360^{\circ}$ in $4^{\circ}$ steps for 5 rotations at 4 min per rotation.

\section{Patlak-Plot Method}

The Patlak-plot method used manual processing with Xeleris (version 3.0; GE Healthcare). ROIs were drawn manually over the aortic arch and bilaterally over the brain hemispheres on sequential radionuclide angiography images, and a time-activity curve was generated. Then, we determined the brain perfusion index and calculated mean $\mathrm{CBF}$ using regression Equation 1 based on the ${ }^{133} \mathrm{Xe}$ method $(18,19)$ :

$$
\text { mean } \mathrm{CBF}=2.59 \times \mathrm{BPI}+19.8 \text {. }
$$

Eq. 1

\section{BUR Methods}

The BUR-C method used manual processing with Xeleris. A time-activity curve to calculate the area under the curve (AUC) was obtained by manually drawing an ROI over the aortic arch on radionuclide angiography images. The time-activity curve was fitted with the $\gamma$-function. The AUC was divided by the ROI area and converted to counts $/ \mathrm{cm}^{2}$. SPECT images were reconstructed using the manual axis setting with ordered-subset expectation maximization. Reconstruction used 6 subsets, 8 iterations, and a Butterworth filter (order, 8; cutoff frequency, $0.49 \mathrm{cycle} / \mathrm{cm}$ ). Attenuation was
FIGURE 1. The 12 ROls standardized using 3DSRT: callosomarginal, precentral, central, parietal, angular, temporal, posterior cerebral, pericallosal, lenticular nuclear, thalamic, hippocampal, and cerebellar.

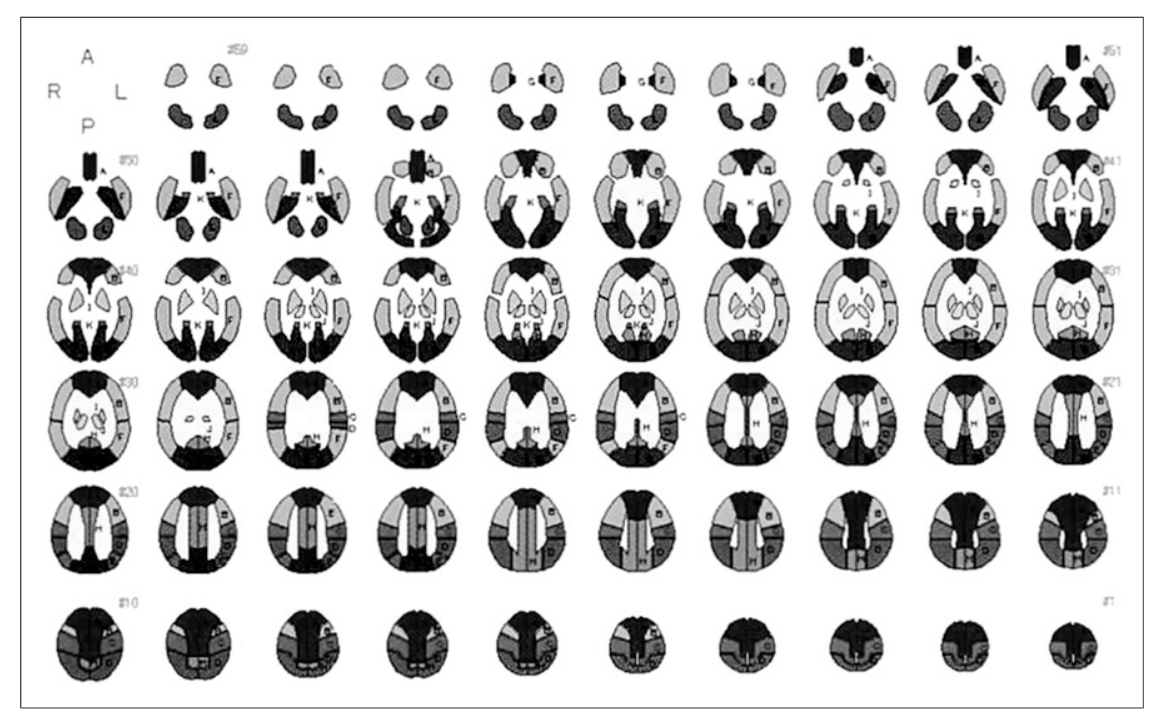




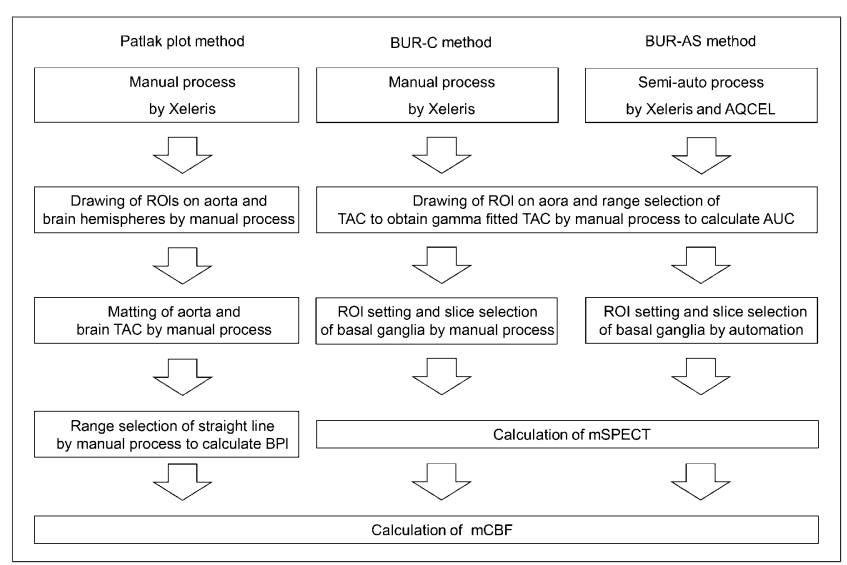

FIGURE 2. Flowchart for Patlak-plot, BUR-C, and BUR-AS methods. Patlak and BUR-C include manual processing. BUR-AS includes manual processing for aortic ROI and time-activity curve range and automatic processing for basal ganglia ROI and slice selection. $\mathrm{mCBF}$ and $\mathrm{mSPECT}=$ mean CBF and SPECT counts, respectively; TAC = timeactivity curve.

corrected using the Chang method (attenuation coefficient, $0.09 \mathrm{~cm}^{-1}$; threshold, 13\%) (20); however, scatter correction was not performed. Basal ganglia slices were selected from transverse images of the manual axis setting, and ROIs to calculate the mean SPECT count were drawn manually on the normal side. Mean SPECT count was calculated using Equation 2:

$$
\text { mean SPECT count }=\frac{\text { SPECT } \text { count in ROI of normal side }}{\text { ROI area (pixels) }} .
$$

The BUR-AS method used semiautomatic processing with Xeleris and AQCEL software (automatic quantitative analysis for cerebral blood flow of ECD tool; Fujifilm RI pharma Co., Ltd.) $(12,13,21)$. AUC was converted to counts $/ \mathrm{cm}^{2}$ using the same process as for the BUR-C method.

The SPECT images were reconstructed by ordered-subset expectation maximization using AQCEL. Image reconstruction and attenuation correction were performed as for the BUR-C method; scatter correction was omitted. Slices $31-40$ of the basal ganglia were selected after anatomic standardization (Fig. 1) (12). The ROIs were automatically set on standardized image slices using a 3-dimensional stereotactic ROI template (3DSRT; Fujifilm RI pharma Co., Ltd.). The ROIs comprised 12 segments (callosomarginal, precentral, central, parietal, angular, temporal, posterior cerebral, pericallosal, lenticular nucleus, thalamus, hippocampus, and cerebellum) (22). The mean SPECT count was calculated from the 3DSRT ROIs of slices 31-40 of the normal-side basal ganglia.

Mean BUR-C and BUR-AS were calculated as in Equation 3 using the AUC-converted count $/ \mathrm{cm}^{2}$ and the mean SPECT count:

$$
\text { mean BUR }=\frac{\text { mean SPECT } \times \mathrm{A}}{\mathrm{AUC}-\text { converted count } / \mathrm{cm}^{2}},
$$

Eq. 3

where A is a cross-calibration factor. The BUR-C and BUR-AS mean $\mathrm{CBFs}$ were calculated using regression Equation 4 based on the ${ }^{123}$ I-IMP microsphere method (9):

$$
\text { mean } \mathrm{CBF}=13.2 \times \text { mean } \mathrm{BUR}^{0.513} .
$$

Figure 2 is a flowchart of the Patlak-plot and BUR-AS methods.

\section{Reproducibility Evaluation}

The Patlak, BUR-C, and BUR-AS mean CBFs were analyzed by 3 radiologic technologists. Inter- and intraoperator reproducibility was estimated using the coefficient of variation (CV) and Bland-Altman plotting, with mean CBF obtained for each of the 3 technologists. To estimate intraoperator reproducibility, the technologists were analyzed 3 times at intervals of more than 1 mo.

\section{Statistical Analysis}

All statistical analyses were performed with EZR (Saitama Medical Center, Jichi Medical University), which is a graphic user interface for $\mathrm{R}$ (version 2.13.0; The $\mathrm{R}$ Foundation for Statistical Computing) (23). The mean CBF CVs were analyzed using the Kruskal-Wallis test, and multiple comparisons among the 3 methods were done with post hoc Steel methodology (the nonparametric analog comparable to the Dunnett test). In all analyses, a $P$ value of less than 0.05 was considered to indicate statistical significance.

\section{RESULTS}

\section{Interoperator Reproducibility}

The CVs of the Patlak, BUR-C, and BUR-AS mean CBFs were $0.039,0.068$, and 0.024 , respectively (Fig. 3). CVs were significantly lower for the BUR-AS method than for the other methods (Patlak vs. BUR-AS, $P=0.001$; BUR-C vs. BUR-AS, $P<0.001)$. Differences in mean $\mathrm{CBF}$ for the 3 methods are shown for each technologist in Figure 4. The average differences in Patlak, BUR-C, and BUR-AS mean CBFs among the 3 technologists were $0.9,1.2$, and -0.8 , respectively. In addition, the limits of agreement were -3.5 to $5.3,-8.1$ to 10.4 , and -3.8 to 2.3 for Patlak, BUR-C, and BUR-AS, respectively. As a result, BUR-AS had the

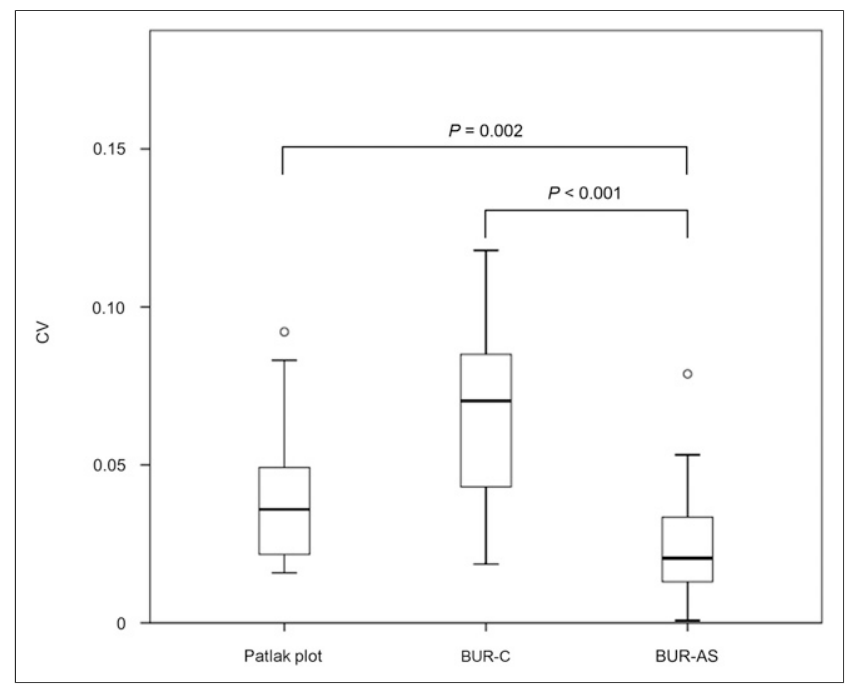

FIGURE 3. CVs for interoperator reproducibility. CV is significantly lowest for BUR-AS. 


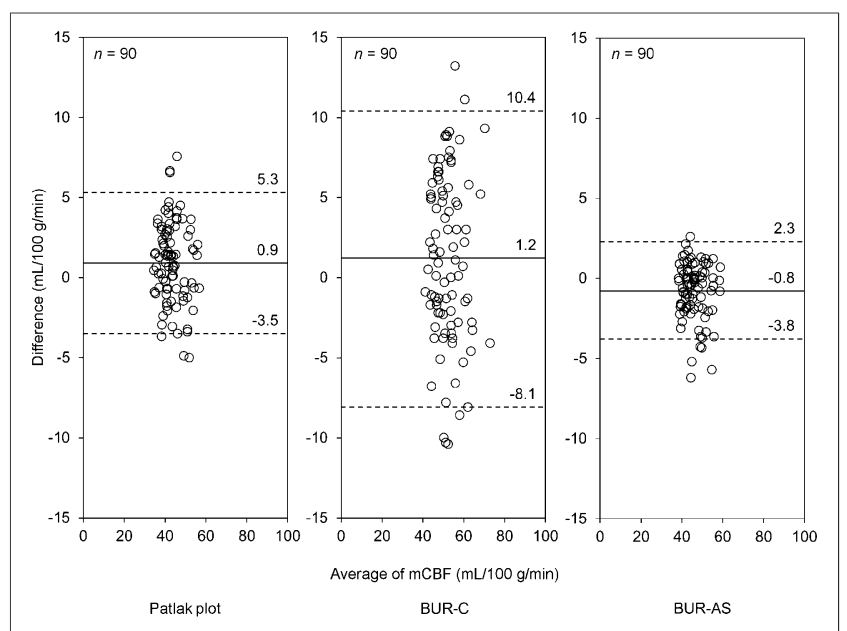

FIGURE 4. Bland-Altman plots for interoperator reproducibility. Difference is lowest for BUR-AS.

smallest mean CBF range of the 3 methods. The case of worst reproducibility among the 3 technologists for the BUR-AS method is shown in Figure 5. The ROIs of the aortic arch, the slice selections for the dynamic images, and the range settings of time-activity curve-fitted $\gamma$-function differed among the 3 technologists.

\section{Intraoperator Reproducibility}

The CVs for the Patlak, BUR-C, and BUR-AS mean CBFs were $0.031,0.024$, and 0.010 for technologist $1 ; 0.028$, 0.020 , and 0.016 for technologist 2 ; and $0.033,0.035$, and 0.022 for technologist 3 (Fig. 6). The BUR-AS method had the lowest mean CBF for all technologists; however, no significant difference was shown between BUR-C and BUR-AS for technologist 2 and between Patlak and BUR-AS for technologist $3(P=0.40$ and 0.057$)$. The difference in each technologist's mean CBF is shown in Figure 7. The average differences in the Patlak, BUR-C, and BUR-AS mean CBFs among the 3 technologists were $0.3,-0.2$, and -0.1 , respectively. In addition, the limits of agreement were -3.7 to $4.4,-4.3$ to 3.9 , and -2.6 to 2.5 for the Patlak-plot, BUR-C, and BUR-AS methods, respectively. As a result, BUR-AS mean $\mathrm{CBF}$ had the smallest range of the 3 methods.

\section{DISCUSSION}

Many institutions have evaluated use of the Patlak-plot and BUR methods to obtain noninvasive quantitative measurements of brain perfusion. Matsuda et al. reported that the CBF obtained from the Patlak-plot method with ${ }^{99 m} \mathrm{Tc}-$ hexamethylpropylene amine oxime converts to ${ }^{133} \mathrm{Xe} \mathrm{CBF}$ using a regression expression (4). They also reported that the Patlak-plot method CBF obtained with ${ }^{99 m}$ Tc-ECD correlates with that obtained with ${ }^{99 \mathrm{~m}} \mathrm{Tc}$-hexamethylpropylene amine oxime (6). Miyazaki et al. reported that the CBF obtained from the BUR method using ${ }^{99 \mathrm{~m}} \mathrm{Tc}$-ECD correlates with that obtained from continuous arterial blood sampling using ${ }^{123}$ I-IMP (9). In clinical studies, Kuroda et al. reported that $\mathrm{CBF}$ and cerebrovascular reactivity derived from quan- titative analysis of stress and rest brain perfusion ${ }^{99 \mathrm{~m} T \mathrm{Tc} \text { SPECT }}$ are useful for diagnosis, staging, and treatment of carotid artery occlusion (24-26). However, Otake et al. reported interoperator differences in the manual setting of ROIs on the aortic arch and bilaterally on the brain hemispheres (11). These differences are considered to influence decisions about treatment and evaluation of revascularization therapy for cerebral ischemia. It is for this reason that we proposed and in this paper have validated the BUR-AS method, which improves inter- and intraoperator reproducibility.

Regarding interoperator reproducibility, the BUR-AS method produced the lowest variability in mean $\mathrm{CBF}$. Although all processing in the Patlak-plot and BUR-C methods was manual, the BUR-AS method used automatic processing except for the drawing of the aortic arch ROI and the fitting of the $\gamma$-function of the time-activity curve. Manual processing reduces reproducibility; the Patlak-plot and BUR-C methods had worse reproducibility than the BUR-AS method. As far as interoperator reproducibility is concerned, BUR-AS was the best of the 3 methods.

Regarding intraoperator reproducibility, the BUR-AS method produced the lowest variability in mean CBF; however, there was no significant difference from the BUR-AS method for BUR-C for technologist 2 and Patlak for technologist 3. One possible reason for the high intraoperator reproducibility of the Patlak-plot and BUR-C methods is the clear criteria that the technologists had. Another possibility is that the BUR-AS method had analysis errors due to the manual processing steps. Stressing the selection criteria would be expected to improve such problems, and automation of all processing would best improve quantitative accuracy. Variability in mean CBF was lowest with the BUR-AS method (Fig. 7), which we therefore suggest to be the best quantitative approach regarding intraoperator reproducibility.

This study had some limitations. First, selection of the aortic arch ROI and fitting of the $\gamma$-function of the timeactivity curve were not automated; the BUR-AS method

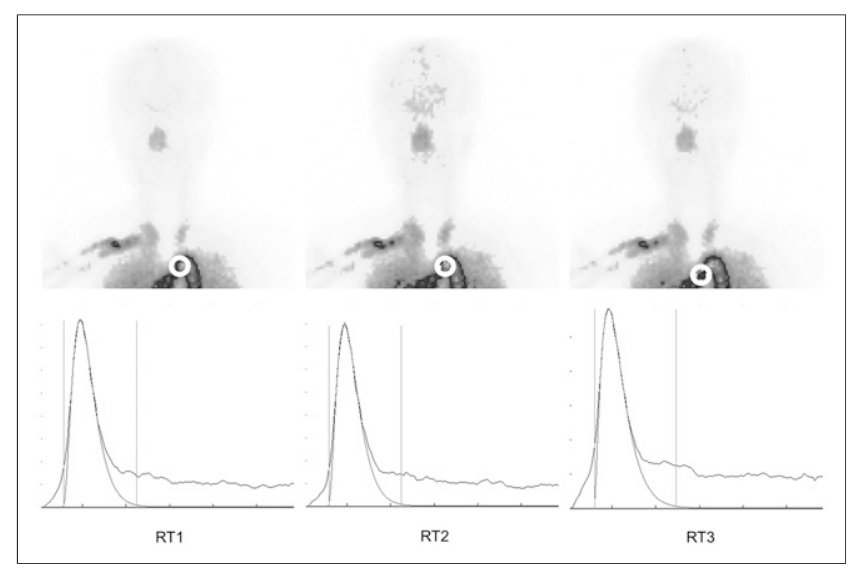

FIGURE 5. Examples of ROI setting and $y$ fitting by the 3 radiologic technologists (RT1, RT2, and $R T 3$ ). At top are the dynamic image slices and the chosen ROI; at bottom are graphs of time-activity curves. 


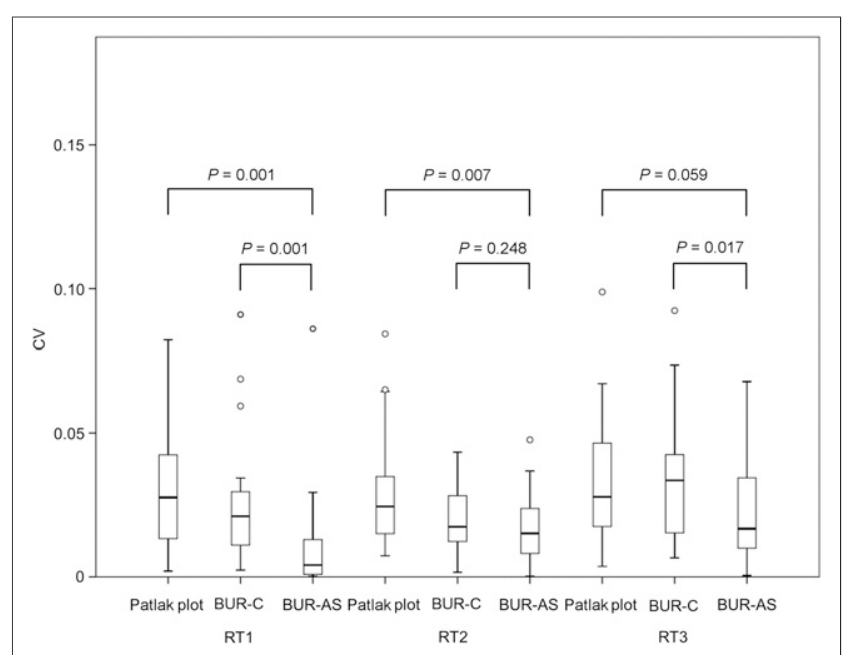

FIGURE 6. CVs for intraoperator reproducibility for the 3 radiologic technologists (RT1, RT2, and RT3). CV was lowest for BUR-AS; CV for BUR-C for RT2 and Patlak plot for RT3 did not significantly differ.

therefore had analysis errors in some cases. Odashima et al. reported improved reproducibility through automation of these functions in the BUR method (17). Use of their method may have improved operator reproducibility in our study. Second, our study used anterior images. Inoue et al. found that using $10^{\circ}$ left anterior oblique images instead of anterior, along with using an ascending aorta ROI instead of aortic arch, gave a good correlation between the BUR method and ${ }^{123}$ I-IMP continuous arterial blood sampling (15). Moreover, Ito et al. made the same alterations and also found a good correlation between the BUR method and $\mathrm{H}_{2}{ }^{15} \mathrm{O} \operatorname{PET}(16)$. Acquisition of the dynamic data in the $10^{\circ}$ left anterior oblique view allows separation of the ascending aorta from the descending aorta, allowing the ROI to easily be set on ascending aorta, and would be expected to improve the inter- and intraoperator reproducibility of the BUR-AS method.

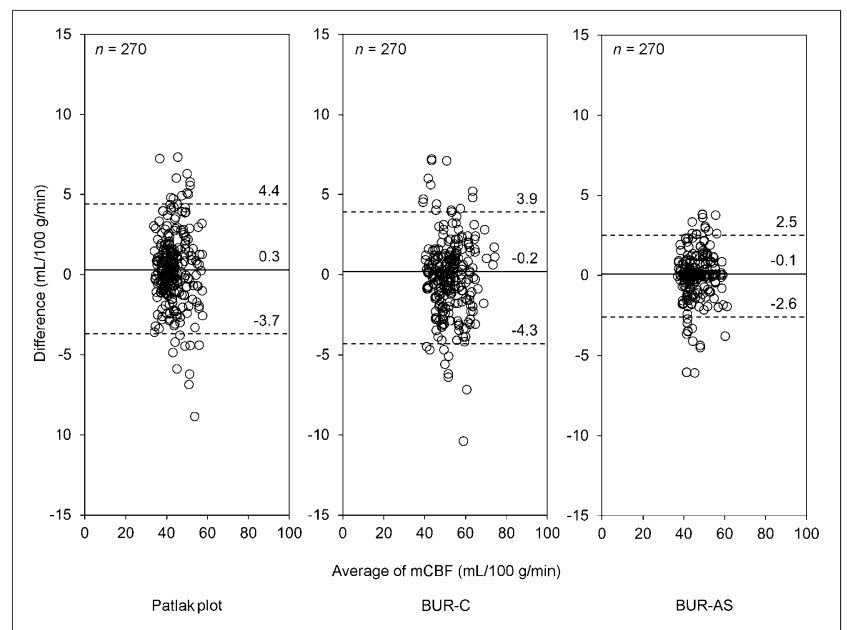

FIGURE 7. Bland-Altman plots for intraoperator reproducibility. Difference is lowest for BUR-AS.

\section{CONCLUSION}

We have demonstrated the inter- and intraoperator reproducibility of mean $\mathrm{CBF}$ as determined using the Patlak-plot, BUR-C, and BUR-AS methods. The BUR-AS method produced the highest reproducibility. Improvement of inter- and intraoperator reproducibility in quantitative analysis of CBF is expected to increase diagnostic accuracy during follow-up and when determining the need for treatment adaptation.

\section{DISCLOSURE}

No potential conflict of interest relevant to this article was reported.

\section{ACKNOWLEDGMENTS}

We thank Akihiro Takaki (Fujifilm RI Pharma Co., Ltd., Tokyo, Japan) for committing to this study. We reported a part of this study at the annual congress of the European Association of Nuclear Medicine, October 21, 2014, Gothenburg, Sweden.

\section{REFERENCES}

1. Nambu K, Suzuki R, Hirakawa K. Cerebral blood flow: measurement with xenon-enhanced dynamic helical CT. Radiology. 1995;195:53-57.

2. Koenig M, Klotz E, Luka B, et al. Perfusion CT of the brain: diagnosis approach for early detection of ischemic stroke. Radiology. 1998;209:8593.

3. Barbier EL, Lamalle L, Decorps M. Methodology of brain perfusion imaging. $J$ Magn Reson Imaging. 2001;13:496-520.

4. Matsuda H, Tsuji S, Shuke N, et al. A quantitative approach to technetium99m hexamethylpropylene amine oxime. Eur J Nucl Med. 1992;19:195200.

5. Matsuda H, Tsuji S, Shuke N, et al. Noninvasive measurements of regional cerebral blood flow using technetium-99m hexamethylpropylene amine oxime. Eur J Nucl Med. 1993;20:391-401.

6. Matsuda H, Yagishita A, Tsuji S, et al. A quantitative approach to technetium99m ethyl cysteinate dimer: a comparison with technetium-99m hexamethylpropylene amine oxime. Eur J Nucl Med. 1995;22:633-637.

7. Miyazaki Y, Kinuya S, Tonami N. A simple method to obtain semiquantitative index of brain perfusion using ${ }^{99 \mathrm{~m}}$ Tc-HMPAO. Kaku Igaku. 1994;31: 551-558.

8. Miyazaki Y, Kinuya S, Hashimoto M, et al. Brain uptake ratio as an index of cerebral blood flow obtained with ${ }^{99 m}$ Tc-ECD. Kaku Igaku. 1997;34:49-52.

9. Miyazaki Y, Mizuta Y, Honda K, et al. Assessment of a new non-invasive cerebral blood flow determination method using the brain uptake ratio of 99m Tc-ECD. Kaku Igaku. 1999;36:113-120.

10. Lassen NA, Andersen AR, Friberg L, et al. The retention of [ $\left.{ }^{99 \mathrm{~m}} \mathrm{Tc}\right]-\mathrm{d}, 1-\mathrm{HM}-\mathrm{PAO}$ in the human brain after intracarotid bolus injection: a kinetic analysis. $J$ Cereb Blood Flow Metab. 1988;8:S13-S22.

11. Otake H, Ujita K, Matsubara K, et al. Multicenter trial for the standardization of mean cerebral blood flow (mCBF) measured by ${ }^{99 m}$ Tc-ECD Patlak plot method. Japanese J Nucl Med Technol. 2003;23:473-476.

12. Takaki A, Yoshioka K, Teraoka S, et al. Improvement in accuracy of quantitative assessment of the regional cerebral blood flow with ${ }^{99 \mathrm{~m}}$ Tc-ECD. Kaku Igaku. 2005;42:11-16.

13. Takaki A, Yoshioka K, Teraoka S, et al. Development of an automatic analytical tool for quantitative cerebral blood flow measurement using ${ }^{99 \mathrm{~m}} \mathrm{Tc}-\mathrm{ECD}$, and verification of clinical examples. Nihon Hoshasen Gijutsu Gakkai Zasshi. 2006;62:729-733.

14. Takaki A, Okada K, Urata J, et al. Technical investigation of cerebral blood flow measurements using the Patlak plot method: a contrivance for positioning of the gamma camera at data collection in radionuclide angiography. Kaku Igaku. 1999;36:139-144. 
15. Inoue S, Kabuto T, Masunaga S, et al. Determination of optimal ROI setting position of the input function for the ${ }^{99 \mathrm{~m}} \mathrm{Tc}$-ethyl cysteinate dimmer brain uptake ratio method. Nihon Hoshasen Gijutsu Gakkai Zasshi. 2012;68:269276.

16. Ito $\mathrm{S}$, Takaki A, Inoue $\mathrm{S}$, et al. Improvement of the ${ }^{99 \mathrm{~m}} \mathrm{Tc}-\mathrm{ECD}$ brain uptake ratio (BUR) method for measurement of cerebral blood flow. Ann Nucl Med. 2012;26: 351-358.

17. Odashima S. New method for improved repeatability of ROI position in aorta, and automation of gamma fitting in BUR examination for non-invasive cerebral blood flow measurement. Nihon Hoshasen Gijutsu Gakkai Zasshi. 2003;59: 1561-1567.

18. Kanno I, Lassen NA. Two methods for calculating regional cerebral blood flow from emission computed tomography of inert gas concentrations. J Comput Assist Tomogr. 1979;3:71-76.

19. Nakano S, Asada T, Matsuda H, et al. Effects of healthy aging on the regional cerebral blood flow measurements using ${ }^{99 \mathrm{~m}}$ Tc-ECD SPECT assessed with statistical parametric mapping. Nippon Ronen Igakkai Zasshi. 2000; 37:49-55.

20. Chang LT. A method for attenuation correction in radionuclide computed tomography. IEEE Trans Nucl Sci. 1978;25:638-643.
21. Asao K, Takeda H, Takaki A, et al. Evaluation of the OS-EM parameter with automatic quantitative analysis for cerebral blood flow of the ECD tool. Nihon Hoshasen Gijutsu Gakkai Zasshi. 2008;64:822-831.

22. Takeuchi R, Yonekura Y, Matsuda H, et al. Usefulness of a three-dimensional stereotaxic ROI template on anatomically standardised ${ }^{99 \mathrm{~m}}$ Tc-ECD SPET. Eur $J$ Nucl Med Mol Imaging. 2002;29:331-341.

23. Kanda $Y$. Investigation of the freely available easy-to-use software 'EZR' for medical statistics. Bone Marrow Transplant. 2013;48: 452-458.

24. Kuroda S, Takigawa S, Kamiyama H, et al. Diagnosis of hemodynamic compromise in patients with chronic cerebral ischemia; the detection of impaired vasodilatory capacity with ${ }^{133} \mathrm{Xe}$ SPECT and acetazolamide (Diamox) test. No Shinkei Geka. 1990;18:167-173.

25. Kuroda S, Kamiyama $\mathrm{H}$, Abe $\mathrm{H}$, et al. Acetazolamide test in detecting reduced cerebral perfusion reserve and predicting long-term prognosis in patients with internal carotid artery occlusion. Neurosurgery. 1993;32: 912-918.

26. Kuroda S, Houkin K, Kamiyama H, et al. Long-term prognosis of medically treated patients with internal carotid or middle cerebral artery occlusion: can acetazolamide test predict it? Stroke. 2001;32:2110-2116. 